birth. Mean scores for the 1-h castrated males and females are presented in Table 2. Although the RQ score is equivalent in both groups, other measures of femininity are consistently lower in 1-h castrated males than in control females.

\section{DISCUSSION}

In the present study, total receptivity scores of the 1-, 6-, and 12-h castrated Ss were averaged to provide an estimate of a group gonadectomized within $24 \mathrm{~h}$ after birth. The mean RQ for such a 1-day castrated group in the present study was .397 (mean of five sessions). For 24-h castrated groups receiving identical amounts of estradiol benzoate, which was $6.6 \mu \mathrm{g}$, the RQ was .280 (mean of four sessions) in Gerall et al's study, and .572(one session of five tests) in Grady et al's experiment. In the present and Gerall et al's study, mean RQs and receptivity scores of 24 -h castrated males were significantly lower than those obtained by control females.

Comparisons among different studies of the effect of gonadectomy within 1 day of birth are, however, of limited value because it is difficult to determine for at least two reasons the actual age of the organism at the time of operation. First, surgery could have occurred at any time within a $24-\mathrm{h}$ period. Secondly, hour of birth is usually identified as when $\mathrm{E}$, who might make one to three inspections per day, first finds the mother with her complete litter. As indicated in Table 1, actual age of individual Ss could differ considerably from age assigned by this method. Data from previous studies, therefore, represent degrees of feminization of a population whose assigned age could vary from actual age up to $24 \mathrm{~h}$ and differences among findings might be related to the total number of Ss castrated fewer than $6 \mathrm{~h}$ after birth.

Data from the present study also raise the question as to whether complete feminization is produced in the 1 -h castrated male.

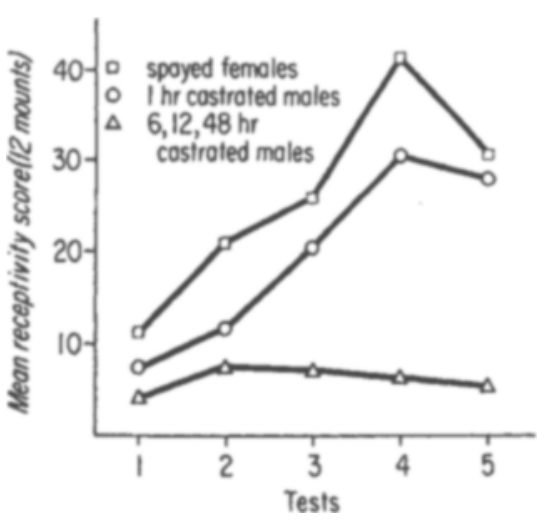

Fig. 2. Mean total receptivity scores obtained in each session by spayed females, 1-h castrated males, and combined 6-, 12-, and 48-h castrated males.

Table 2

Receptivity Scores and Quotients of 1-h Castrated Males and Spayed Females

\begin{tabular}{lccccc}
\hline & \multicolumn{3}{c}{ Receptivity Score } & \multirow{2}{*}{ Receptivity Quotient } \\
\cline { 1 - 3 } Groups & Total & Lordosis & Holding & Darting & \\
\hline Spayed female & 26.9 & 16.2 & 6.3 & 4.4 & .691 \\
1-h castrated male & 20.0 & 14.8 & 2.7 & 2.5 & .680 \\
\hline
\end{tabular}

The RQ score provides an affirmative, whereas the holding measure, a negative answer. Differences in darting also suggest that complete feminization has not been accomplished. It might be suggested that the lower degree of holding in the 1 - $h$ group is due to the absence of a vagina preventing the male's intromission pattern with its rapid dismount. Holding may have been less apparent following the slow dismount of an incomplete intromission. However, since a female given estrogen and progesterone will exhibit lordosis and hold in the absence of intromissions and even of full mounts, the difference between the females and 1 -h castrated males on this measure probably represents true female behavior capacities. Although males castrated $1 \mathrm{~h}$ after birth could not be statistically distinguished from spayed females by either the mean RQ or the total qualitative receptivity score, the conclusion that postnatal castration effects complete feminization of the male must wait for future studies. The holding measure, as noted above, was different between the two groups and there was a general tendency for frequency of darting and quality of lordosis to be lower in the males. Also, only one level of estrogen was used in the tests and lower dosages will have to be studied before a general conclusion is reached.

\section{REFERENCES}

GERALL, A. A., HENDRICKS, S.E., JOHNSON, L. L., \& BOUNDS, T. W. Effects of early castration in male rats on adult sexual behavior. Journal of Comparative \& Physiological Psychology, 1967, 64, 206-212.

GRADY, K. L., PHOENIX, C. H., \& YOUNG, W. C. Role of the developing rat testis in differentiation of the neural tissues mediating mating behavior. Joumal of Comparative \& Phy siological Psychology, 1965, 59, 176-182. NOTES

1. This study was supported by Research Grant HD 00867-07 from the National Institute of Child Health and Human Development, United States Public Health Service.

2. Estradiol benzoate was generously supplied by the Schering Corporation.

\title{
Cortical polarization effects on consolidation of avoidance learning in rats
}

\author{
STEPHEN D. BERRY, DAVID G. SIM, and \\ CHARLES W. SNYDER, University of \\ Notre Dame, Notre Dame, Ind. 46556
}

Anodal and cathodal cortical polarization, previously hypothesized to be effective in influencing learning consolidation, was given to 18 rats following a learning task. Results showed significant difference in performance as a function of the direction of current flow.
Rusinov (1953), using anodal polarization in the motor cortex of the rabbit, found a "dominant focus of excitation"-a nonspecific hypersensitivity of a response to any sensory stimulation. This, he felt, demonstrated that extracellular current fields exerting an electronic influence on cell populations play a definite role in the cellular interactions within the brain. Morrell (1961), using rabbits and cats, found the same nonspecific hypersensitivity during 


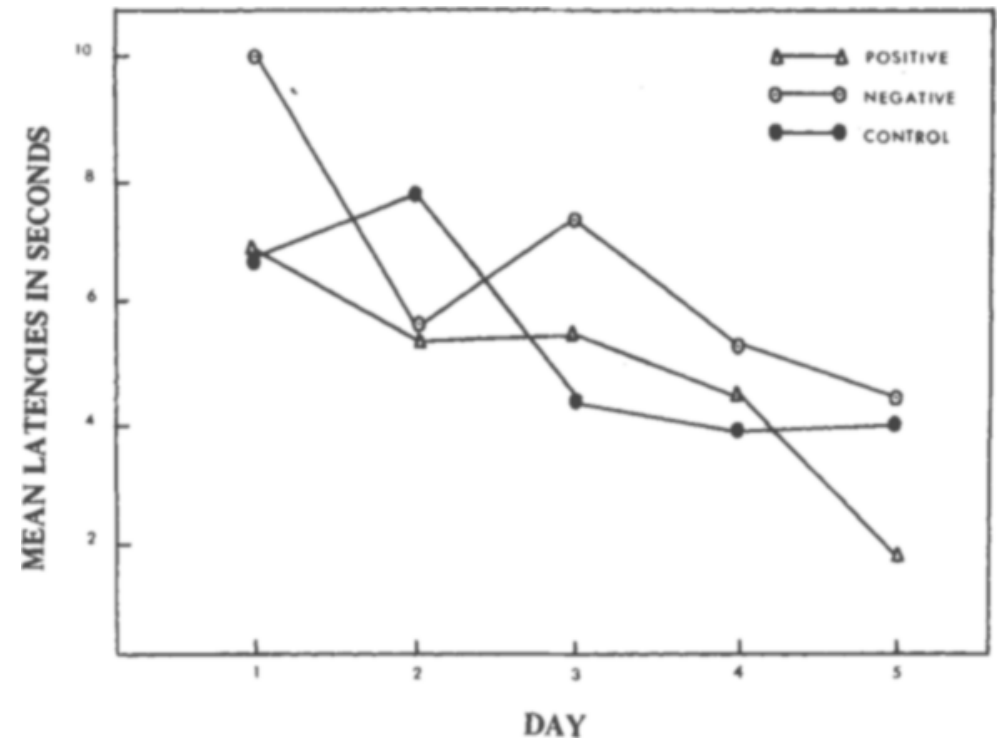

Fig. 1. Group mean latencies in test trials on Days 1-5.

anodal polarization. However, he noted that after cessation of polarizing current only those stimuli which had been presented during polarization elicited the appropriate response. Albert found that cathodal polarization can prevent interhemispheric transfer (1966b), and anodal polarization can restart consolidation of an interhemispheric transfer (1966a) originally prevented by potassium spreading depression in rats. His results therefore suggested that the consolidation of learning requires electrical potential gradients in the cortex of the brain, and that consolidation might be disturbed by manipulating this potential.

In the present experiment polarization was given following each daily training trial on a one-way shuttle escape-avoidance task. Three independent groups received either anodal, cathodal, or no polarization, under the hypothesis that anodal current would result in more efficient consolidation and cathodal current less than in the control condition.

\section{METHOD}

The Ss were 18 naive, male, albino rats, weighing 250-390 g, and 90-120 day s of age at the beginning of testing.

The apparatus was a one-way shuttlebox ( $4.5 \mathrm{ft} \times 6$ in. 7 in.). Two feet of the length composed a white-walled, grid-floored chamber, separated by a sliding door from the black-walled goal box, $2.5 \mathrm{ft}$ in length. A variable autotransformer provided shock current through a 20,000-ohm resistor and grid scrambler. Polarizing current was supplied by a Grass S-5 stimulator, providing an output of 50 microamperes of either anodal or cathodal polarizing current. Two timers and a capacitor-resistor chargedischarge circuit automatically produced a continuous recycling of a unidirectional pulsating current. Thirty-six stainless steel electrodes and 36 2-mm o.d. polyethylene cannulae containing a $0.9 \%$ saline solution were used.

The Ss were divided into three groups of six members each. Group 1 received anodal polarizing current, Group 2 cathodal polarizing current, and Group 3 no current. The members of each group were anesthetized, and two stainless-steel electrodes with cannulae attached were implanted on the cortex of each rat. The electrodes were located $2.5 \mathrm{~mm}$ on either side of the midsaggital suture and $3.5 \mathrm{~mm}$ posterior to bregma. A stainless-steel electrode for the return current was implanted in the back of each rat. The control group underwent this procedure in order to control for variations in performance attributable to anesthesia and postoperative effects.

Approximately 5 days after surgery, the testing began. Each rat in Group 1 was treated as follows: The $\mathrm{S}$ was placed in the shuttlebox, starting the timer. After 5 sec the shock was presented at a level of $100 \mathrm{~V}$ and a strength of $2.5 \mathrm{~mA}$, and was terminated when the $S$ moved into the goal box. The intertrial interval was on a VI 30 -sec schedule with intervals ranging from 15 to $45 \mathrm{sec}$. Each session consisted of a test trial and a learning trial. The $E$ recorded the latency between the start of the trial and the response.

After each session, the $S$ was returned to a special holding chamber and immediately given $1 \mathrm{~h}$ of slowly pulsating anodal polarizing current. The current was given in $2-\mathrm{min}$ cycles of $15 \mathrm{sec}$ increase from 0.0 to 50.0 microamperes, $45 \mathrm{sec}$ of steady 50.0-microampere current, 15 -sec decrease from 50.0 to 0.0 microamperes, and $45 \mathrm{sec}$ at 0.0 microamperes. Each $S$ underwent six sessions, at 24-h intervals, receiving anodal polarizing current under the same procedure after each session.

This procedure was followed exactly for Group 2 and Group 3, with variations in the type of polarization current. Cathodal current was given to Group 2, while Group 3 was given no current, although the members of this group were placed in the polarizing chambers.

\section{RESULTS AND DISCUSSION}

The data recorded were the latencies between the start of the trial and the exhibition of the appropriate avoidance or escape response. The mean group response on test trials across days was calculated. These 18 scores were used in an analysis of variance for a simple randomized design, determining differential effects of treatment. Significant results were obtained from a $t$ test run between the positive and negative group test results $(t=4.09, d f=5$, $p<.001)$. Neither the analysis of variance nor further $t$ tests proved significant.

The results indicated that, although treatment effects were not significantly different from the control, polarization had an effect on leaming beyond chance levels. Comparison of the two treatment effects showed that manipulation of the direction of polarization reliably influenced learning. The learning effect within each treatment and the relationship among the three treatments over trials are illustrated in Fig. 1.

As the graph shows, there is a highly reliable difference in performance between positive and negative groups. The lack of reliable differences from the control group can be partly accounted for by the extreme variability within that group in each session.

The significance of these data indicates that polarization can have an influence on learning without the use of potassium spreading depression to delay the onset of consolidation. In addition, data from a pilot study and a subsequent study now in progress suggest that strength of polarization is an important parameter. The potential usefulness of polarization warrants further investigation of this area.

\section{REFERENCES}

ALBERT, D. J. The effect of spreading depression on the consolidation of learning. Neuropsychologia, 1966a, 4, 49-64.

ALBERT, D. J. The effects of polarizing currents on the consolidation of learning. Neuropsychologia, 1966b, 4, 65-76.

MORRELL, F. Effect of anodal polarization on the firing pattern of single cortical cells. Annals of the New York Academy of Science, 1961, 92, 860-876.

RUSINOV, V. S. An electrophysiological analy sis of the connecting function on the cerebral cortex in the presence of a dominant region area. Abstract Communications, XIX International Physiological Congress, Montreal, 1953,719-720. 